\title{
Leserbefragung
}

\section{Was tun Ärzte bei Atemwegsinfekten?}

\author{
In Deutschland kommen zu häufig Antibiotika zum Einsatz. Schuld sind angeblich die \\ Ärzte in der Primärversorgung, die diese zu unkritisch verschreiben. Eine Umfrage unter \\ Allgemeinmedizinern zeigt jedoch, dass bei Atemwegsinfekten an erster Stelle Phyto- \\ therapeutika verordnet werden. Die Ergebnisse sind auch für Kinderärzte relevant.
}

W ährend der Erkältungszeit kommen die meisten Patienten wegen Atemwegsinfektionen zum Arzt. In der Hälfte der Praxen sind es $25-50 \%$ der Patienten, in jeder fünften Praxis sind es sogar über $50 \%$. Diese Quoten ergeben sich aus der Leserumfrage „Antibiotika-Resistenzen - wie vermeiden?", die Springer Medizin mit Unterstützung des Unternehmens Bionorica SE bei Allgemeinärzten durchgeführt hat.

\section{Örtliche Resistenzsituation kennen}

Die Befragung ergab außerdem, dass rund $30 \%$ der Teilnehmer nicht gut über die Resistenzsituation vor Ort beziehungsweise in der Region Bescheid wissen. Andere informieren sich bei ihrem Mikrobiologielabor (37\%), beim RobertKoch-Institut (23\%), bei örtlichen Kliniken oder dem Gesundheitsamt (jeweils knapp $10 \%)$ und den Referenzzentren (6\%).

Dabei ist das Wissen um die regionale und lokale Resistenzsituation eine wichtige Voraussetzung, um bei einer bakteriellen Infektion zum richtigen Antibiotikum zu greifen. Das Mikrobiologielabor, mit dem eine Praxis zusammenarbeitet, sollte die erste Anlaufstelle für Infos zur Resistenzsituation vor Ort sein. Auch die Antibiotika Resistenz Surveillance (ars) am Robert-Koch-Institut ist eine geeignete Informationsquelle.

\section{Einsatz von Bedarfsrezepten}

Viele Ärzte befürworten Bedarfsrezepte für Antibiotika. In der Praxis werden diese aber kaum genutzt. Jeder zweite Teilnehmer (47\%) der Umfrage der „Ärzte Zeitung“ tut dies nur selten, mehr als jeder vierte nie (27\%). $14 \%$ gaben an, häufig Bedarfsrezepte zu nutzen, aber nur knapp 5\% tun dies regelmäßig. Dabei zeigt die Erfahrung, dass ein solches Rezept geeignet ist, den Patienten mit Atemwegsinfekten zusätzlich Sicherheit zu geben. Es dient als Rückversicherung für den Patienten oder im Falle von Kindern, für die Eltern. So ist bereits nach dem ersten Arztbesuch ein Rezept verfügbar, falls das Labor (Blutbild, BSG, CRP) doch auf einen bakteriellen Infekt hinweist. Auch wenn „red flags“ auftreten, wie etwa steigendes Fieber oder Husten mit starken Schmerzen hinter dem Brustbein, kann das Rezept eingelöst werden.

\section{Atemweginfekte und Antibiotika}

Der Patientenwunsch nach einem Antibiotikum wird meist nicht erfüllt: Nur $11 \%$ der Ärzte gaben an, bei Atemwegsinfekten unkritisch ein Antibiotikum zu verordnen (Abb. 1). Zwei Drittel raten dagegen zu pflanzlichen Arzneimitteln. Phytopharmaka werden auch in einigen der knapp 30 AWMF-
Leitlinien, die es zu Erkältungskrankheiten gibt, empfohlen. Dies sind vor allem Thymian, Efeu und Myrtol, bei Halsschmerzen auch Salbei. Ihr Vorteil sind eine große therapeutische Breite bei geringen Nebenwirkungen. Denn die Vielstoffgemische setzen an mehreren Zielstrukturen an und haben unterschiedliche Wirkungen.

Werden Antibiotika verordnet, erfolgt dies gezielt: $80 \%$ verschreiben sie ihren Risikopatienten, zum Beispiel mit Immunschwäche. Auch bei schwerer Symptomatik über einen längeren Zeitraum greifen $71 \%$ zum Antibiotikum. Bei Patienten mit grünlich-gelbem Auswurf sind es $38 \%$. Die Sputumfarbe ist aber kein geeigneter Parameter, um virale und bakterielle Infektionen $\mathrm{zu}$ unterscheiden. In einer Studie hatte nur jeder sechste Patient mit gelblich-grünlichem Auswurf einen bakteriellen Infekt (Altiner A et al. Scand J Prim Health Care 2009; 27:70-3).

Dr. Michael Hubert

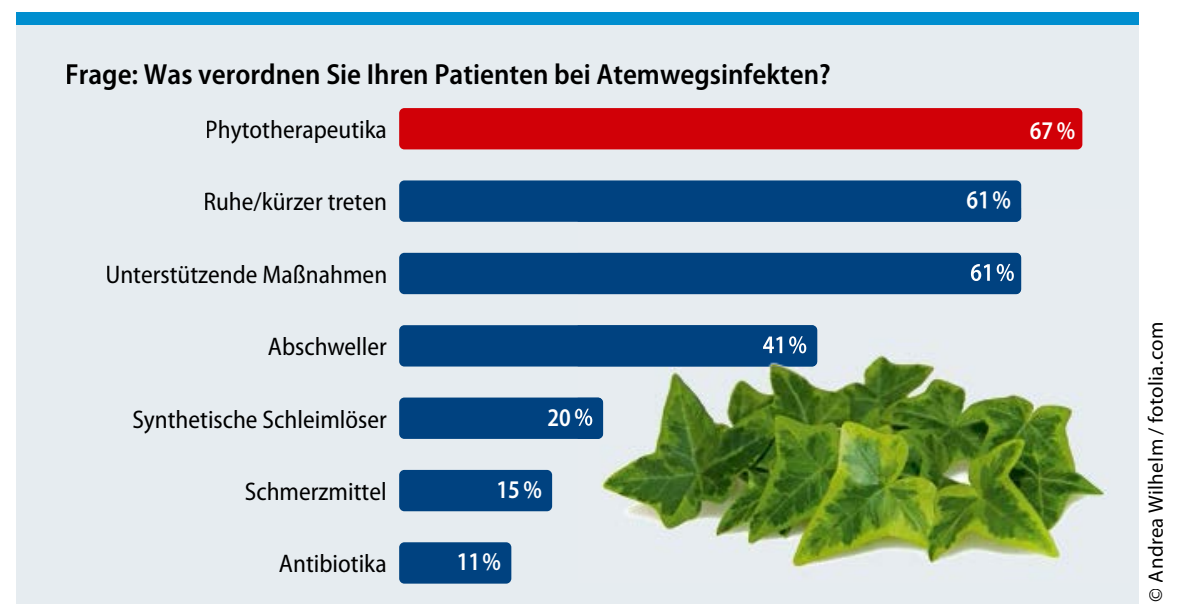

Abb. 1: Ergebnis der Leserumfrage von Springer Medizin: Phytotherapeutika sind für viele Ärzte das Mittel der Wahl bei Atemwegsinfekten 